\title{
Entscheiden trotz Nichtwissen
}

\section{Das Beispiel der Sanierung kontaminierter Flächen}

\author{
Von Alena Bleicher ${ }^{1}$
}

Zusammenfassung: Entscheidungen der Umweltgestaltung müssen mit der Herausforderung umgehen, dass umfassendes Wissen über Prozesse der natürlichen Umwelt und die Auswirkungen menschlicher Eingriffe häufig nicht vorliegt. Dass diese Situation nicht, wie häufig angenommen, zu Unsicherheiten im Entscheiden führen muss, zeigt das Beispiel der Sanierung kontaminierter Flächen. Akteure, die in Sanierungsprojekte involviert sind, setzen sich offenbar explizit mit dem Unbekannten und seiner Handlungsrelevanz für Entscheidungen auseinander und finden Strategien für den Umgang mit überraschenden Entwicklungen. Diese Feststellung ist anschlussfähig an Desiderata der jüngeren sozialwissenschaftlichen Nichtwissensforschung: neben der wissenschaftlichen Wissensbeschaffung als Grundlage von Entscheidungen sollten weitere Strategien im Umgang mit unbeantworteten Fragen und Nichtwissen berücksichtigt werden. Wenig untersucht ist bisher, wie solche alternativen Strategien in alltäglichen Entscheidungsprozessen aussehen können. Basierend auf einem dynamischen Konzept des Nichtwissens wird im vorliegenden Artikel gezeigt, wie Akteure die Handlungsrelevanz von Nichtwissen bestimmen. Ausgehend von der Analyse von zwei Projekten der Altlastensanierung wird ein analytisches Modell von Entscheidungen entwickelt, in die Nichtwissen aktiv einbezogen wird. Elemente wie die Begründbarkeit von Entscheidungen, das Verständnis von Fehlern und das Vorbereitetsein auf Überraschungen sind ebenso Teile des Modells wie die Kompensationsstrategien im Umgang mit unvorhergesehenen Ereignissen. Auf diese Weise wird gezielt ein Handlungsrahmen geschaffen, der einen offenen Umgang mit Nichtwissen erlaubt.

\section{Einleitung - Die Unvollständigkeit des Wissens}

Die Gesellschaften westlicher Industriestaaten sind seit Beginn des 20. Jahrhunderts von der Vorstellung geprägt, wissenschaftliches Wissen könne umfassende Erklärungen für Prozesse und Zusammenhänge in der Natur liefern und damit Entscheidungen sicherer machen. Nur selten sind jedoch Entwicklungen genau vorherzusehen, sodass den Möglichkeiten der Umweltgestaltung Grenzen gesetzt sind. Angesichts der Komplexität von Prozessen und Wechselwirkungen zwischen Gesellschaft und Ökosystemen wird mehr und mehr anerkannt, dass auch die Wissenschaft nicht in der Lage ist, vollständige und endgültig gesicherte Erklärungen zu liefern. Wissenschaftliche Erklärungen sind vielmehr häufig widersprüchlich und neues Wissen wirft immer auch neue Fragen auf (vgl. Lau 1989; Faber et al. 1993; Smithson 1993; Dovers et al. 2001; Liskog 2011). Entschieden werden muss dennoch und es stellt sich die Frage, wie vor diesem Hintergrund sichere Entscheidungen getroffen werden können.

Ein Themenfeld, in dem der Unvollständigkeit des Wissens im Entscheidungsmoment von den Akteuren offenbar eine gewisse Normalität eingeräumt wird, ist die Sanierung kontaminierter Flächen. In einem Handbuch zur Altlastensanierung schreibt der Ingenieur Edmund Brandt (1993: 14): „Typisch für die Altlastendiskussion ist, dass ständig neue Problemfelder sichtbar werden, sich insgesamt die Fragestellung ausweitet und Bereiche einzubeziehen sind, die zuvor nicht für einschlägig erachtet wurden.“

Flächen, auf denen sich durch industrielle Produktionen in der Vergangenheit Schadstoffe anreicherten, werden als Altlastenflächen bezeichnet und sind in allen industrialisierten Ländern zu finden. In den westlichen Industrieländern entstanden sie in der ersten Hälfte des 20. Jahrhunderts, als die negativen Wirkungen bestimmter Substanzen auf Ökosysteme und die

1 Ich danke den beiden anonymen Gutachtern für ihre wertvollen Hinweise zum Manuskript

Soziale Welt 63 (2012), S. $97-115$ 
menschliche Gesundheit noch nicht bekannt waren und Restprodukte der Industrieproduktion ohne besondere Sicherheitsvorkehrungen abgelagert wurden oder durch Havarien in Boden und Grundwasser gelangten. Aufgrund des fehlenden Problembewusstseins wurden diese Handlungen nur selten dokumentiert und das Wissen über die Ablagerungen geriet in vielen Fällen in Vergessenheit. Ende der 1970er Jahre wurden die Gefahren dieser sogenannten Altlasten erkannt und es entwickelte sich die Altlastensanierung als Themenfeld des nachsorgenden Umweltschutzes. Während bis zur Mitte der 1980er Jahre die Abwehr von Gefahren für Mensch und Umwelt im Vordergrund stand, rückte seitdem die Etablierung neuer urbaner Nutzungen auf Industriebrachen (z.B. Hafengeländen und innerstädtische Industriegeländen) ins Zentrum der Aufmerksamkeit. Diese Wiedernutzung wurde ein attraktiver Beitrag zur Verringerung des Flächenverbrauchs und der nachhaltigen Stadtentwicklung (vgl. Dixon et al. 2007; De Sousa 2008; Franzius et al. 2009). Im Zusammenhang mit diesen Flächenentwicklungen ist immer auch die Beseitigung von Altlasten notwendig.

Der Umgang mit Altlasten ist durch die ständige Auseinandersetzung mit dem Unbekannten gekennzeichnet. Zusätzlich zur fehlenden Dokumentation über Art und Umfang der Ablagerungen, verändern die im Boden und Grundwasser vorhandenen Schadstoffe im Lauf der Zeit ihre chemische Struktur (Zersetzung durch Umwelteinflüsse) und verbreiten sich ausgehend vom Ort ihrer Ablagerung oft auch auf angrenzende Flächen. Die Erfahrung zeigt, dass trotz sorgfältiger Erkundung nicht alle Details hinsichtlich der Zusammensetzung des ,Schadstoffcocktails" und seiner räumlichen Verteilung auf einer Fläche vorab bestimmt werden können. Entscheidungen müssen häufig getroffen werden, obwohl noch kein umfassendes Wissen vorliegt.

Zur Beschreibung solcher Entscheidungen etablierte sich in den 1970er Jahren der Begriff des Risikos. Allerdings wurde recht schnell in Teilen der sozialwissenschaftlichen Umweltforschung mit dem Verweis auf die Erklärungsgrenzen des Risikobegriffs die Einführung der Kategorie „Nichtwissen“ (ignorance) vorgeschlagen, um das eigentlich zentrale Phänomen in der sogenannten Risikogesellschaft - die prinzipielle Unvollständigkeit des Wissens über die Prozesse der natürlichen Umwelt - hervorzuheben (vgl. Collingridge 1980; Beck et al. 1996; Green 2009). ${ }^{2}$ Indem das Nichtwissen ins Zentrum der Betrachtung gerückt wird, so die Annahme, kann der Frage nachgegangen werden, wie sich Gesellschaften mit den Grenzen des Wissens auseinandersetzen und welche Konstruktionsprozesse stattfinden. Außerdem kann eine Auseinandersetzung sowohl mit den Handlungsmöglichkeiten angesichts unbeantworteter Fragen als auch mit den Strategien im Umgang mit nicht vorhersehbaren Ereignissen stattfinden (vgl. Smithson 1985; Stocking 1998).

Anknüpfend an die Desiderata der sozialwissenschaftlichen Nichtwissensforschung beschäftigt sich der vorliegende Artikel mit der Relevanz des Nichtwissens in alltäglichen Entscheidungen. Am Beispiel von Entscheidungsprozessen in der Altlastensanierung wird den Fragen nachgegangen, wie Akteure mit Nichtwissensentscheidungen umgehen und welche Kompensationsstrategien sie entwickeln.

\section{Die Allgegenwart des Nichtwissens in der Gesellschaft}

Im Zentrum der seit den 1990er Jahren intensivierten soziologischen Nichtwissensforschung steht bisher das wissenschaftliche Nichtwissen, das Ausgangspunkt und Anreiz für Forschungsarbeiten und Innovationen ist. Die Vorstellung eines von Robert K. Merton als spezifiziert bezeichneten Nichtwissens (specified ignorance) ist dabei zentral (Merton 1987). Erst wenn ein Wissen über das Nichtwissen besteht, können Forschungsbedarfe benannt werden

2 Zur Diskussion der Grenzen des Risikobegriffs vergleiche die ausführliche Darstellung bei Wehling 2011. 
sowie Entwicklung und Lernen stattfinden. Nichtwissen ist damit die Vorbedingung für das Kerngeschäft der Wissenschaft. Autoren wie Robert N. Proctor oder Scott Frickel u.a. beschäftigten sich mit der Frage, wie genau die Formulierung relevanter wissenschaftlicher Fragestellungen stattfindet und welchen Einflüssen sie unterliegt (Proctor 1995; Frickel et al. 2010). Proctor arbeitete am Beispiel der Krebsforschung in den USA und Deutschland heraus, wie Forschungsprioritäten, Machtverhältnisse, Ideologien und Interessen innerhalb und auBerhalb der Wissenschaft die Agenden wissenschaftlicher Forschung beeinflussen (Proctor 1995). Nichtwissen, so die Erkenntnis, kann genau wie Wissen als strategische Ressource dienen und unterliegt ähnlichen Konstruktionsprozessen (Stocking 1998; McGoey 2007). Andere Autoren identifizierten darüber hinaus strukturelle Ursachen für die Erzeugung von Nichtwissen, die systematisch in wissenschaftlichen Curricula und Arbeitsweisen angelegt sind. Dazu gehören z.B. die Selektivität wissenschaftlicher Theorien, die Art und Weise der Konstitution unbekannter Wirkungshorizonte oder der Vorgang der Dekontextualisierung experimentell erzeugten Wissens (Ravetz 1993). Durch die Definition und Abgrenzung von Forschungsthemen werden also einerseits Nichtwissensansprüche definiert und gleichzeitig andere Themen (bewusst oder unbewusst) ausgeblendet und nicht bearbeitet.

Allerdings ist es selten der Wissenschaft alleine vorbehalten, forschungsrelevante Wissenslücken zu definieren. Vielmehr sind Politik, Medien und Gesellschaft an der Definition von Nichtwissens-Ansprüchen beteiligt und fordern diese Teilhabe ein. Einerseits können aus Sicht nichtwissenschaftlicher Akteure Fragestellungen eine hohe Relevanz haben, die von der Wissenschaft nicht bearbeitet werden. Andererseits können definierte Forschungsfragen von der Allgemeinheit als Bedrohung wahrgenommen werden, wie beispielsweise die Debatte um das Recht auf Nichtwissen im Zusammenhang mit genetischen Diagnosen deutlich macht (Wehling 2006). Verschiedene Autoren beschäftigen sich mit den Mechanismen der Nichtwissenskonstruktion im Zusammenspiel von Wissenschaft und gesellschaftlichen Akteuren und fragen nach der Rolle und dem Einfluss institutioneller Konstellationen (vgl. z.B. Smithson 1993; Stocking / Holstein 1993; Hess 2009; de Krom / Oosterveer 2010).

Mit dem Herauslösen der Nichtwissensdefinition aus dem rein wissenschaftlichen Kontext wird sie zur gesamtgesellschaftlichen Herausforderung und Verantwortung und es muss geklärt werden, wie dieser Prozess in die Gesellschaft eingebettet werden kann. Diese Frage ist in die Forschungen zur partizipativen oder post-normalen Wissenschaft eingebettet, die sich mit der Gestaltung des Verhältnisses zwischen wissenschaftlicher Forschung und Gesellschaft auseinandersetzen (vgl. z.B. Funtowicz / Ravetz 1993; Gibbons et al. 1994). In diesem Zusammenhang kommt ein weiterer Aspekt des Nichtwissens zum Tragen: das Problem der Ungleichheit von Wissensressourcen. So wird davon ausgegangen, dass Laien Zugang zu spezifischen (lokalen) Wissensbeständen haben, auf die Forscher nicht ohne weiteres zurückgreifen können. Diese Wissensbestände, so das zentrale Argument in entsprechenden Forschungsarbeiten, sollten in die wissenschaftliche Wissensproduktion einbezogen werden (vgl. Collins / Evans 2002; Hess 2009; Steyaert / Jiggins 2007; Powell / Colin 2009).

So unterschiedlich diese Forschungsperspektiven sind, so fällt doch auf, dass in diesen Arbeiten Nichtwissen fast ausschließlich in seiner Relation zu Wissen verstanden wird. Nichtwissen ist das, was nicht gewusst wird - unvollständiges Wissen oder noch-nicht-Wissen. Es wird als das Aufzulösende verstanden. Die Suche nach Strategien im Umgang mit Nichtwissen bleibt in diesen Perspektiven folglich auf die Generierung von Wissen fokussiert, wie z.B. das Bemühen um immer präzisere Prognosemodelle und Szenarien oder die Konstruktion von Werkzeugen zur Bewertung von Entscheidungsfolgen zeigen.

Eine weitere Perspektive auf Nichtwissen findet sich bereits in der frühen soziologischen Beschäftigung mit Nichtwissen. Es wird angenommen, dass Nichtwissen und Wissen gleichermaßen eine gesellschaftserhaltende Funktion haben. Georg Simmel stellte in seinen Über- 
legungen über „Das Geheimnis und die geheime Gesellschaft“ heraus, dass Nichtwissen ebenso wie Wissen Grundlage menschlichen Handelns sind und in dieses bewusst einbezogen werden. Er verdeutlicht das „Spiel“ mit Wissen und Nichtwissen anhand der Entstehung von Geheimnissen in der Moderne durch den Prozess der Individualisierung. Geheimnisse führen zur Entstehung sozialer Gruppen, die einen ganz bestimmten Wissensschatz (Faktenwissen, aber auch Wissen über bestimmte Rituale und Verhaltensweisen) hüten (Simmel 1908; Gross 2012). Der Zugang zu diesem Wissen wird bewusst exklusiv gestaltet, erzeugt ein Zusammengehörigkeitsgefühl innerhalb der Gruppe und ist Distinktionsmerkmal gegenüber anderen sozialen Gruppen (z.B. Experten vs. Laien). Nichtwissen wird in dieser Perspektive als stabilisierendes Element des gesellschaftlichen Zusammenhalts gesehen. Alltagsweltliche Phänomene wie Privatheit und Höflichkeit wären ohne Nichtwissen ebenso wenig möglich wie bestimmte soziale Situationen, die ihren Reiz gerade durch die Unvollständigkeit des Wissens erhalten (z.B. Glücksspiele in Spielkasinos oder das erste Rendezvous) (Moore / Tumin 1949; Smithson 1985).

Nichtwissen in seiner gesellschaftserhaltenden Funktion und als ein dem Wissen gleichberechtigtes Phänomen anzuerkennen scheint gerade dann hilfreich zu sein, wenn es darum geht, Strategien und institutionelle Arrangements zu identifizieren, die den Umgang mit Nichtwissen jenseits der Generierung von Wissen ermöglichen.

Eine Nichtwissenstypologie, die auf der Annahme basiert, dass Nichtwissen gesellschaftskonstituierend und für Handlungen relevant ist, wurde von M. Groß vorgeschlagen (2007, 2010). Ausgehend von dem Grundverständnis, dass Nichtwissen ein spezifizierbares Nichtwissen ist, also dass es möglich ist, ein Wissen darüber zu haben was nicht gewusst wird, führt er fünf Begriffe ein: ignorance ${ }^{3}$ (das unspezifische Nichtwissen über einen Sachverhalt), nonknowledge (handlungsrelevantes Nichtwissen), negative-knowledge (nicht handlungsrelevantes Nichtwissen), extended-knowledge (Wissen als Ergebnis der Wissenserarbeitung) und nescience (nicht reflektierbares Nichtwissen). Der Übergang zwischen diesen Nichtwissensformen erfolgt jeweils durch eine bewusste Auseinandersetzung mit dem Nichtwissen und durch die Entscheidung über seine Handlungsrelevanz. In der Reflexion vergangener Ereignisse können Akteure feststellen, dass sie nichtwissend waren, ohne dass sie dieses Nichtwissen hätten reflektieren können (nescience). Mit dem Bewusstwerden dieses Nichtwissens handelt es sich bereits um (unspezifisch) reflektiertes Nichtwissen, das in Entscheidungsprozesse einbezogen werden kann (ignorance). Akteure können darüber verhandeln, welche Bedeutung dem Nichtwissen in anstehenden Handlungen zukommt. Wird Nichtwissen für den weiteren Handlungsverlauf als wichtig erachtet, handelt es sich um handlungsrelevantes Nichtwissen (non-knowledge), wird es als unwichtig erachtet, wird von nicht handlungsrelevantem Nichtwissen gesprochen (negative-knowledge). Erfolgt eine Auseinandersetzung mit Nichtwissen kann neues Wissen entstehen (extended-knowledge).

Statt also lediglich eine bestimmte Qualität von Nichtwissen (z.B. vollständig, teilweise, unauflöslich etc.) zu benennen, werden in dieser Terminologie die aktive Beschäftigung mit dem Unbekannten und die Veränderung seiner Handlungsrelevanz für handelnde Akteure in den Vordergrund gestellt. Im Gegensatz zur Linearität von möglicherweise etablierteren Typologien erlaubt es dieser Ansatz weiterhin, Verknüpfungen zwischen den einzelnen Formen des Nichtwissens herzustellen und die Übergänge zwischen ihnen zu erklären.

Um die Relevanz von Nichtwissen und ihre Veränderung in Entscheidungsprozessen der Revitalisierung altindustrieller Flächen aufzuspüren, braucht es eine Typologie, die bewusste

3 Angesichts der Vielfalt existierender Nichtwissens-Terminologien im Deutschen und Englischen werden die originalen Begriffe hier wiedergegeben und im Folgenden verwendet. Damit ihre Klarheit erhalten bleibt. 
Entscheidungen von Akteuren im Umgang mit Nichtwissen zum zentralen Bezugspunkt macht und damit grundsätzlich die Existenzberechtigung von Nichtwissen anerkennt. Aus diesem Grund stützen sich die im Folgenden dargestellten Analysen von Altlastensanierungen auf diese Typologie.

\section{Methodischer Ansatz}

Die Auseinandersetzung von Akteuren mit Nichtwissen und die Entwicklung von Kompensationsstrategien in alltäglichen Entscheidungsprozessen der Altlastensanierung wurden auf Grundlage von zwei empirischen Beispielen analysiert. Eine der gewählten Fallstudien war ein Sanierungsprojekt in der Gemeinde Weißandt-Gölzau im deutschen Bundesland SachsenAnhalt, das über 1,5 Jahre begleitet wurde. Die zweite Fallstudie war eine Altlastensanierung in der Stadt Troutdale im US-Bundesstaat Oregon, die im Rahmen eines dreiwöchigen Forschungsaufenthaltes untersucht wurde. In Weißandt-Gölzau erfolgte die Altlastensanierung im Rahmen der Erneuerung der Verkehrsinfrastruktur im örtlichen Industriegebiet, die von der Gemeinde initiiert und in ihrem Auftrag in Kooperation mit zahlreichen Akteuren durchgeführt wurde. Die Bodenbelastungen, in erster Linie verschiedene Kohlenwasserstoffe, gingen auf die Anfangszeit der Industrialisierung in den 1920er Jahren zurück. Zu dieser Zeit wurde auf dem Gelände des heutigen Industriegebietes Braunkohle abgebaut und an Ort und Stelle zu Kraftstoffen und Ölen raffiniert. Obwohl es seit den 1990er Jahren ein Wissen über die möglichen Kontaminationen gab und auch Erkundungen durchgeführt wurden, kam es während des Sanierungsprojektes (2005-2007) immer wieder zu unerwarteten Altlastenfunden, mit denen die beteiligten Akteure umgehen mussten.

Auf dem ehemaligen Fabrikgelände in Troutdale gingen die Kontaminationen auf die Aluminiumproduktion seit den 1940er Jahren zurück. Restprodukte wurden auf dem Firmengelände ungesichert abgelagert, was zur Belastung des Bodens und des Oberflächenwassers mit Fluorid und Schwermetallen führte. Bereits acht Jahre vor der Werksschließung im Jahr 2002 wurde auf gemeinsame Initiative der US-amerikanischen Umweltbehörde US EPA und des produzierenden Unternehmens Reynolds Metals begonnen, die Schadstoffablagerungen auf dem Gelände zu erkunden und zu sanieren. Trotz sorgfältiger Voruntersuchungen wurden immer wieder unerwartete Altlasten gefunden. Es wird davon ausgegangen, dass auch nach dem Ende der Sanierung im Rahmen künftiger Bauaktivitäten zur Ansiedlung neuer Nutzungen mit nicht entdeckten Altlasten zu rechnen ist.

Basierend auf leitfadengestützten Interviews mit insgesamt 26 Projektbeteiligten - Gemeindevertretern, Umweltbehörden, Investoren und Ingenieuren - wurden für beide Fallstudien Entscheidungsprozesse rekonstruiert und der Umgang der Akteure mit Nichtwissen analysiert. Neben den mündlichen Darstellungen wurden auch schriftliche Dokumentationen der Entscheidungen wie Verträge, Gemeinderatsprotokolle und Gutachten in die Analyse einbezogen.

In diesem empirischen Material wurde Nichtwissen auf verschiedene Weise deutlich: Schlüsselbegriffe verwiesen auf Wissensgrenzen („Das hat keiner gewusst" oder „...irgendwie wird es sich einregeln..."), auf die Zukunft gerichtete Erwartungshaltungen beinhalteten Wissenslücken (,Wir erwarten eigentlich noch kontaminiertes Material...") und offene Fragen wurden genannt („Wie stark ist das Grundwasser kontaminiert?“). ${ }^{4}$ Häufig ließ sich der Umgang mit Nichtwissen aus der Darstellung von Handlungsfolgen rekonstruieren. Wurden z.B. Erkundungsbohrungen durchgeführt, konnte geschlussfolgert werden, dass die Akteure mit Nichtwissen konfrontiert waren, ihm Handlungsrelevanz zuschrieben (non-knowledge) und entsprechende Maßnahmen der Auseinandersetzung ergriffen. In bestimmten Zusammenhän-

4 Erste sprachwissenschaftliche Auseinandersetzungen mit Ausdrucksformen von Nichtwissen in Texten finden sich bei Stocking und Holstein (1993), Kewell (2010) sowie Janich et al. (2010). 
gen ist die Darstellung des Nichtwissens offensichtlich bereits institutionalisiert. So müssen z.B. Flächen, auf denen Altlasten vermutet werden, aber präzises Wissen fehlt, als sogenannte Altlastenverdachtsflächen, im Flächennutzungsplan durch eine Signatur gekennzeichnet werden. Auch in diesem Fall wird dem Nichtwissen eine Handlungsrelevanz zugewiesen.

Methodisch orientierte sich die Analyse des empirischen Materials am Ansatz der Grounded Theory (Strauss / Corbin 1990; Strübing 2008). Interviews und Dokumente wurden digitalisiert und mit Hilfe des Computerprogramms MAXQDA analysiert (vgl. Kuckartz 2007). Ausgehend von der systematischen Analyse der Interviews und Dokumente wurden wiederkehrende Themen identifiziert, in mehreren Durchläufen zu Oberthemen zusammengefasst und auf diese Weise kodiert. Nach der Zusammenfassung der Kodierungen zu Konzepten erfolgte in weiteren Auswertungsschritten die Erarbeitung ihrer Eigenschaften und ihrer Zusammenhänge. Schließlich wurde auf dieser Grundlage ein Modell entwickelt, das die Aspekte darstellt, die Nichtwissensentscheidungen ermöglichen, und (erste) Hypothesen über ihre Zusammenhänge formuliert.

\section{Die Relevanz von Nichtwissen in Altlastensanierungen}

Die Tatsache, dass Wissensgrenzen in den Fallstudien im Rahmen von Interviews und Dokumenten teilweise sehr präzise benannt werden, zeigt, dass grundsätzlich ein großes Bewusstsein über unbeantwortete Fragen hinsichtlich der Altlastensituation besteht. Die Thematisierung dieses Nichtwissens und seiner Relevanz werden von den Interessen der Akteure, von ihrer Rolle im Projekt und von ihrem jeweiligen Zugang zu spezifischen Wissensressourcen beeinflusst. So hat ein Flächeneigentümer häufig wenig Interesse daran, genau zu wissen, ob und wie der Boden und das Grundwasser belastet sind, da damit für ihn Verpflichtungen und finanzielle Aufwände entstehen können. Gerade wenn ein Grundstück verkauft werden soll, wird ein Eigentümer versuchen, Nichtwissen als nicht relevant darzustellen. Demgegenüber ist es das zentrale Geschäftsfeld eines Ingenieurbüros, offene Fragen als Grundlage für Untersuchungen zu formulieren. Demzufolge besteht ein Interesse daran, sich mit Nichtwissen auseinanderzusetzen.

Entsprechend waren es auch in den Fallbeispielen die Experten aus Ingenieurbüros und Fachverwaltungen, die unbeantwortete Fragen hinsichtlich der Altlastensituation thematisierten und Vorschläge zum Umgang mit Nichtwissen in Diskussionen unterbreiteten. Diese Rolle wurde ihnen auch von anderen Beteiligten zugewiesen, wie ein Lokalpolitiker zum Ausdruck brachte:

„Die Gemeinde hält sich eigentlich aus diesem Geschehen, sag ich mal, von den Entscheidungen heraus [...] weil der fachliche Verstand einfach nicht da ist, muss man sagen. Die Fachleute sitzen auf der Seite und denen sollte man das überlassen. " (Lokalpolitiker, 2007)

Gleichwohl blieben Entscheidungen über den Umgang mit Kontaminationen nicht allein Experten überlassen. Die Rekonstruktion der Fallstudien zeigte, dass von Experten identifizierte Wissenslücken auf regelmäßigen Projekttreffen (Bauberatungen) in die Diskussionen eingebracht und auf diese Weise auch mit den Nicht-Experten geteilt wurden. So konnte eine gemeinsame Auseinandersetzung über die Relevanz des Nichtwissens für die Entscheidungsfindung und nachfolgende Handlungen stattfinden.

Nicht relevant konnte Nichtwissen beispielsweise aus Sicht vo Investoren sein, wenn diese vor der Realisierung ihrer Projekte auf den Flächen Altlastenerkundungen durchführen mussten:

„Zu große Sicherheiten [in der Altlastenerkundung] einzubauen führt zu dem Ergebnis, dass der Geldgeber unter Umständen vollkommen verschreckt wird, für die Maßnahme. 
Meistens wollen sie es gar nicht hören. ,Wir fangen mal an und dann schauen wir weiter ' “ (Ingenieur, 2008)

Dieser Darstellung zufolge wurden offene Fragen durch den Ingenieur zwar identifiziert, aber vom Investor als nicht relevant für die folgenden Handlungen und Entscheidungen angesehen (negative-knowledge). Wie dieses Beispiel verdeutlicht, wird die Relevanz des Nichtwissens offenbar im Dialog verhandelt: Ingenieure weisen auf aus ihrer Sicht relevante Fragen hin. Der Investor versucht mit Blick auf die durch Untersuchungen zu erwartenden Kosten die Anzahl dieser Fragen zu minimieren. Der sich hier andeutende Konflikt unterschiedlicher Relevanz von Nichtwissen für einzelne Akteure wird nicht nur in den analysierten Beispielen durch eine dialogische Annäherung der Parteien gelöst. Fragen, die im Hinblick auf eine Gefahrenvermeidung zu beantworten sind, werden durch die Umweltbehörde definiert und lassen wenig Verhandlungsspielraum. Bei Fragestellungen, die über behördliche Erfordernisse hinausgehen, entscheidet letztendlich der Investor als Geldgeber, welchen Fragen nachgegangen wird und welche Untersuchungen vor Baubeginn durchgeführt werden. Unabhängig davon, ob Altlasten vorab identifiziert werden oder erst im Zuge von Bauarbeiten auftreten, muss ihre Sanierung durch den Investor finanziert werden. Gleichwohl erstellen Ingenieure häufig eine Rangfolge der Fragen nach ihrer Dringlichkeit, die sich einerseits aus den gesetzlichen Erfordernissen und andererseits aus dem Erfahrungswissen des Ingenieurs über die jeweiligen Kontaminanten ergibt. Auf dieser Grundlage wird dann entschieden, welches Nichtwissen im Moment der Entscheidung relevant und welches nicht relevant ist.

Die Möglichkeit, offene Fragen zu einem späteren Zeitpunkt wieder aufzugreifen, wird dabei aktiv in Betracht gezogen, wie im letzten Satz des vorangegangenen Zitats angedeutet wird. Als nicht relevant erachtetes Nichtwissen kann durch weitere Entwicklungen plötzlich relevant werden und überraschende Ereignisse können neue Erkenntnisse bringen, die vorangegangene Untersuchungen in anderem Licht erscheinen lassen und weitere Untersuchungen zur gleichen Frage nötig machen. So wurde z.B. in Weißandt-Gölzau in den 1990er Jahren unter dem Industriegelände eine ungewöhnlich hohe Akkumulation von Methangas festgestellt. Eine genaue Ursachenforschung und die Abschätzung einer möglichen Explosionsgefahr wurden in dieser Zeit jedoch nicht als relevant angesehen. Erst vor Beginn der Straßensanierung im Jahr 2005, die auch bis zu diesem Zeitpunkt nicht genutzte Flächen berührte, wurde dieses Nichtwissen handlungsrelevant, so dass auch diesen offenen Fragen nachgegangen wurde.

In vielen Situationen kamen Akteure überein, dass Nichtwissen handlungsrelevant ist und Antworten vor der Entscheidung gefunden werden mussten: Nichtwissen (ignorance) wurde relevant (non-knowledge) und in der Beschäftigung mit dem Nichtwissen wurde erweitertes Wissen (extended-knowledge) geschaffen. Mit Hilfe technischer (Erkundungs-) Methoden konnte ein Überblick über die Altlastensituation auf der jeweiligen Fläche und damit Wissen geschaffen werden, wie ein Behördenvertreter schilderte:

„Dann wird auf dieser Fläche eine isolierte Untersuchung durchgeführt. Also, wie ist der Untergrund beschaffen? Das bündelt sich in einem Bericht. Der sagt also hier ist so und so viel Stoff mit Teer, mit dieser Zusammensetzung, mit diesem Belastungsszenario. “ (Fachbehörde, 2007)

$\mathrm{Zu}$ Beginn einer Altlastenerkundung stehen in aller Regel konkrete Fragen, die mit Hilfe neu erworbenen Wissens beantwortet werden sollen. Aussagen zu Schadstoffgruppen und ihrer Verteilung sowie zu Gefährdungspotenzialen können auf Grundlage dieser Untersuchungen getroffen werden. Allerdings kann auch erweitertes Wissen immer wieder auf unbeantwortete Fragen und Nichtwissen hinweisen, über dessen Handlungsrelevanz entschieden werden muss. Altlastenerkundungen dauern aus diesem Grund häufig mehrere Jahre. 
Das Wissen um diesen Prozess und die Erfahrung, dass trotz sorgfältigster Erkundung nicht alle Schadstoffe identifiziert und lokalisiert werden können, führt dazu, dass auch Entscheidungen getroffen werden, obwohl das als wichtig erachtete Wissen noch nicht geschaffen wurde. Die Auseinandersetzung mit dem Nichtwissen erfolgt dann häufig parallel zu Sanierungs- und Konstruktionsarbeiten. Eine Vertreterin der US-amerikanischen Umweltbehörde (US EPA) stellte eine entsprechende Situation wie folgt dar:

„When we did this investigation, which took about four years to complete, meanwhile these removal actions were going on. So there was clean up work going on. " (Bundesbehörde, 2010)

Die Akteure handelten offensichtlich bewusst nicht auf der Grundlage umfassenden Wissens und nahmen mögliche unvorhergesehene Ereignisse in Kauf.

Auch in der Altlastensanierung gibt es Situationen, in denen der Verweis auf Nichtwissen der beispielsweise von Stocking (1998) beschriebenen Rechtfertigung der Abwesenheit von Wissen oder auch der eigenen Entlastung und der Abgabe von Verantwortung dient. In den beiden Fallstudien fand sich diese Strategie in der Verhandlung über die Handlungsrelevanz von Nichtwissen aber nicht wieder. Die Auseinandersetzung mit der Bedeutung von Nichtwissen war hier aktiv auf weitere Handlungen und nicht auf ihre Vermeidung gerichtet.

Die Fragen, wie überhaupt Entscheidungen trotz Nichtwissen getroffen werden können und wodurch sich diese Entscheidungsprozesse auszeichnen, bildeten den Ausgangspunkt für ein analytisches Modell von Nichtwissensentscheidungen. Das auf Basis der Fallstudien entwickelte Modell stellt die Aspekte dar, die in Entscheidungsprozessen eine Rolle spielen, in die Nichtwissen aktiv einbezogen wird.

\section{Der Umgang mit Nichtwissen - ein analytisches Modell}

Die Auseinandersetzung mit der Handlungsrelevanz von Nichtwissen bildet den Ausgangspunkt des Modells (vgl. Abb. 1). Sie wird von der Begründbarkeit einer Entscheidung sowie von einem veränderten Verständnis von Fehlern (und in der Folge dem Verständnis von Haftung) ermöglicht und wirkt wiederum auf diese zurück. Die Thematisierung von Nichtwissen führt dazu, dass Akteure auf unvorhersehbare Ereignisse eingestellt sind (Zustand des Vorbereitetseins) und Handlungen mit größerer Aufmerksamkeit ausführen. Auf Grundlage dieses Vorbereitetseins können schließlich konkrete Kompensationsstrategien für den Umgang mit unvorhergesehenen Ereignissen entwickelt werden. 
Abbildung 1: Analytisches Modell von Entscheidungen unter Einbeziehung von Nichtwissen (Entwurf Bleicher)

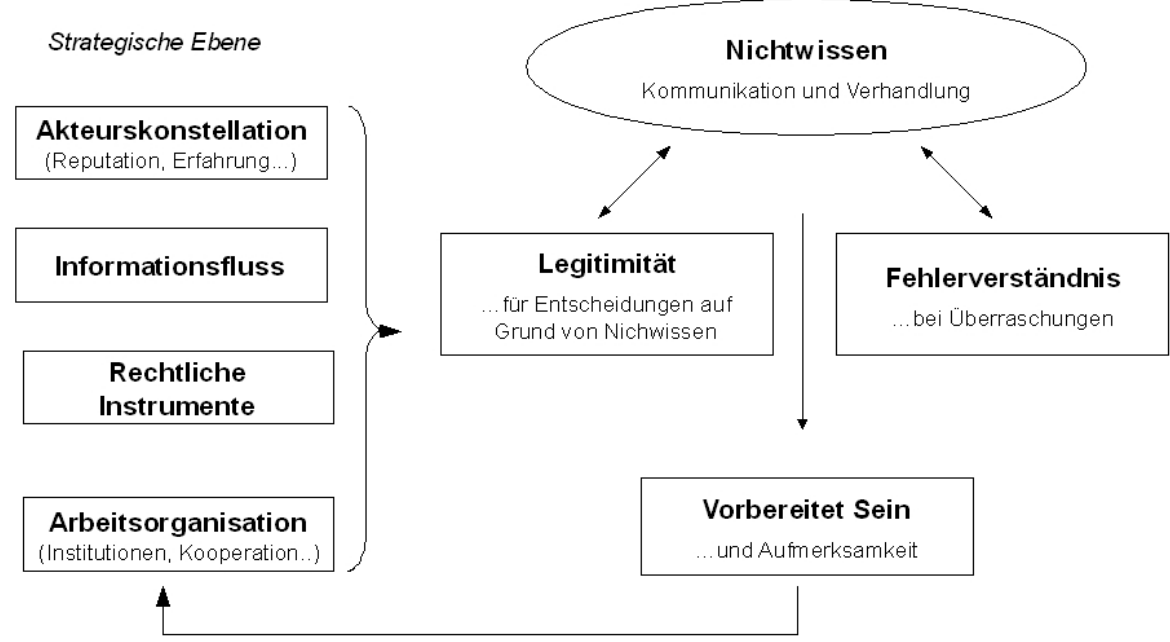

\section{Gute Gründe für Nichtwissensentscheidungen}

Entscheidungen müssen begründet werden. Nicht immer ist die tatsächliche Begründung im Moment der Entscheidung reflektiert, häufig wird sie erst im Nachhinein bewusst gemacht. Empirisch überprüfbares, wissenschaftlich-rationales Wissen ist in modernen Gesellschaften eine entscheidende und allgemein akzeptierte Begründung für Entscheidungen (Weingart et al. 2007; Hofer 2009). Steht wie im Fall von Nichtwissensentscheidungen kein umfassendes Wissen zur Verfügung, so müssen andere von den Beteiligten akzeptierte Begründungen gefunden werden. In den Fallstudien wurden vier entsprechende Begründungsmuster identifiziert.

Zum Ersten kann mit dem Verweis auf ökonomische Vernunft entschieden werden, beispielsweise wenn die Kosten für eine weitere Wissensgewinnung den erwarteten Nutzen deutlich übersteigen. Ein Ingenieur stellte diese häufig gewählte Begründung wie folgt dar:

, [...] meistens ist es ja so, dass Auftraggeber in die Erkundung kein Geld reinstecken wollen. Weil, das kostet alles nur Geld und das reicht mir [an Wissen]. [...] Ich mach ein Erkundungskonzept und der Auftraggeber sagt: ,Ach nee, die Bohrung, die bezahl ich euch nicht'. “ (Ingenieur, 2008)

Auch wenn die Empfehlung des Experten ist, weiteres Wissen zu schaffen, kann mit dem Hinweis auf die möglicherweise überflüssigen finanziellen Aufwendungen für ein Handeln trotz Nichtwissen entschieden werden. Nichtwissen wird in diesem Fall als nicht handlungsrelevant gesehen (negative-knowledge). Obwohl dieses Beispiel auch als (vielleicht sogar unverantwortliche) Sparmaßnahme interpretiert werden kann, weisen die weiteren Ausführungen des zitierten Ingenieurs darauf hin, dass der Entscheidung eine Abwägung des Investors zwischen erhofftem Wissensgewinn und später eventuell notwendig werdenden Investitionen für Sanierungsmaßnahmen zugrunde liegt. Aaron Wildavsky argumentierte bereits in den 1980er Jahren, dass es nicht sinnvoll ist, Ressourcen ausschließlich in die Schaffung sicher geglaubten 
Wissens zu investieren, weil dann unter Umständen im Moment wirklich notwendiger Anpassungen keine Mittel mehr zur Verfügung stehen (Wildavsky 1988). Vor diesem Hintergrund wird in Sanierungsprojekten häufig mit praktischer Orientierung ein Weg zwischen technisch möglicher und für den Moment als ausreichend angesehener Wissensgenerierung gesucht.

Zum Zweiten kann legitim entschieden werden, wenn zu befürchten ist, dass die Schaffung von Wissen geplante Aktivitäten stark verzögert. Nichtwissen wird als handlungsrelevant erachtet, eine Beantwortung der Fragen erfolgt aber parallel zu anderen Aktivitäten. Der folgenden Darstellung eines Behördenvertreters ist zu entnehmen, dass die mit komplizierten Schadensfällen verbundenen Gefährdungsabschätzungen so zeitintensiv sind, dass es als nicht sinnvoll eingeschätzt wird, bereits angelaufene Arbeiten und Planungen zu unterbrechen:

„, Wir können bei einem derartigen Komplex leider [noch] nicht sagen, was diese Gefährdungen tatsächlich für Maßnahmen erfordern. Das bedeutet aber noch nicht, dass ich jetzt, bevor ich diese Gefährdungsabschätzung habe, mit allem anhalten und sagen kann: Wartet mal!" (Fachbehörde, 2007)

Auf die Ergebnisse zu warten wird in der gegebenen Situation als nachteiliger eingeschätzt als trotz bekannter Wissenslücken zu handeln. Diese Begründung wird häufig genannt, da die Erkundung von Altlasten oft Jahre in Anspruch nehmen kann. Die bestehende Situation wird gleichzeitig als so nachteilig eingeschätzt und wahrgenommen, dass scheinbar jede Entwicklung zur Verbesserung der Situation führt, so dass es akzeptiert ist eine Sanierung zu beginnen, bevor die letzten Details erkundet sind.

Der Hinweis auf akuten Handlungsdruck stellt eine dritte Möglichkeit der Begründung dar. Sie wird in erster Linie dann gewählt, wenn während der Durchführung von Sanierungsarbeiten unvorhergesehene Ereignisse eintreten, die mit einer akuten Gefährdung von Mensch und Umwelt verbunden sind. Mit diesen muss unverzüglich umgegangen und müssen ad hoc neue (technische) Lösungen gefunden werden, wie das folgende Zitat illustriert:

„Und mit einem Schlag stellt man fest: Huch, das ist ja sogar im Grundwasser! Und da musste sofort, auf der Stelle eine Grundwasserreinigungsanlage ranorganisiert werden. Das heißt, da muss natürlich mal blitzartig die Strategie gewechselt werden - neue technische Lösungen - und die müssen dann halt auch mal innerhalb von 1-2 Tagen [da sein]. " (Ingenieur, 2007)

Zeit für die Erarbeitung von Wissen ist in diesen Situationen oft nicht vorhanden, und auch die Einbindung in den übergeordneten Sanierungsplan muss hintenan gestellt werden. Weiterführende Fragen, die sich aus dem unvorhergesehenen Ereignis möglicherweise ergeben, werden häufig erst im Nachgang geklärt und in die Fortentwicklung der Sanierungsstrategie einbezogen. Nichtwissen hat demzufolge für diesen Moment keine Handlungsrelevanz, kann diese aber infolge der Ereignisse bekommen.

Ein viertes Begründungsmuster ist die Unberechenbarkeit der Natur, die es unmöglich macht, im konkreten Moment weiteres Wissen zu schaffen. Eine solche Situation kann zum Beispiel durch Eingriffe im Zuge von Sanierungsmaßnahmen entstehen, wie ein Projektmanager herausstellte:

„Das ist ein Riesenloch da hinten, 7500 qm, und alle vorhandenen Wasserschichten sind einfach jetzt durchgehackt worden. Es wird sich irgendwie was dort neu einregeln müssen. Was, weiß keiner. " (Projektmanager, 2007)

Ein solcher Hinweis auf die Unberechenbarkeit der Natur verlagert das Problem des Nichtwissens auf Kausalitäten, die außerhalb der menschlichen Handlungsmöglichkeiten liegen. Für 
die Beteiligten ist es nicht möglich, sich entsprechendes Wissen zu beschaffen, so dass keine andere Wahl bleibt, als abzuwarten und wenn nötig eine Entscheidung zu treffen, die nicht auf fundiertem Wissen basiert.

Die Gültigkeit der dargestellten Begründungsmuster für Nichtwissensentscheidungen ist an bestimmte Situationen im Projektablauf gebunden. Aus diesem Grund werden diese kontextspezifischen Entscheidungsbegründungen teilweise in Verträgen festgeschrieben. Mit Hilfe einer Formulierung wie „nach derzeitigem Kenntnisstand“ wird beispielsweise auf Gutachten und Analysen verwiesen, in denen wiederum konkrete Wissenslücken benannt werden. Im Kontext der Altlastensanierung handelt es sich bei dieser häufig in Verträgen zu findenden Rhetorik demnach nicht nur um einen allgemeinen Verweis auf Nichtwissen, sondern um notwendige Handlungsrichtlinien. Dieses Vorgehen hat den Vorteil, dass die Begründung von Entscheidungen für alle Beteiligten zu jedem Zeitpunkt des Projektes nachzuvollziehen ist. Im Zusammenhang mit dem Verständnis davon, was als ein mit Haftungsfragen verbundener Fehler zu verstehen ist, wird die Bedeutung dieser Festlegungen deutlich.

\section{Ungünstige Entwicklungen müssen keine Fehler sein}

An die Identifizierung von Fehlern und ihrer Zuschreibung auf einen Akteur sind Fragen der Haftung und der Verantwortlichkeit geknüpft (Lau 2009). Fehler gelten als vermeidbar, wenn sorgfältig unter Einhaltung anerkannter Praktiken gehandelt wird. Das folgende Zitat eines Ingenieurs macht den Zusammenhang zwischen vermeidbaren Fehlern und Haftungsfragen deutlich, gibt aber gleichzeitig Hinweise auf das besondere Verständnis von Fehlern in Situationen des Nichtwissens (hier als Unsicherheit bezeichnet) bei der Altlastensanierung.

,Wenn aber an einer Stelle, wo wir nicht erkundet haben, was vollkommen anderes rauskommt, wo uns kein Vorwurf gemacht werden kann, dass wir irgendwie fahrlässig irgendwas übersehen haben, dann ist das halt die Unsicherheit. [...] Es ist nicht ganz so einfach, ein Ingenieurbüro, also jetzt im Altlastenbereich, haftbar zu machen, weil einfach inhärent viele Fehler sind. Also beispielsweise, wenn ich eine Brücke konstruiere und ich verrechne mich und die stürzt ein, ist das ein eindeutiger Fehler. So, wenn ich bei einer Altlastensanierung, bei einer Erkundung, irgendwas nicht erkunde, dann gibt es viele verschiedene Möglichkeiten, warum ich das nicht erkunde. "(Ingenieur, 2008)

Kann ein ungünstiges Ereignis auf eine abweichende, als falsch geltende Anwendung von Wissen zurückgeführt werden (hier eine falsche Berechnung), dann wird dieses Ereignis als (eindeutiger) Fehler interpretiert. Die Identifizierung eines Fehlers bildet dann die Grundlage dafür, dass Fragen von Haftung und Verantwortung durch die Zuschreibung auf einem Akteur geklärt werden können. Die Darstellung des Ingenieurs macht deutlich, dass im Zusammenhang mit der Beseitigung von Kontaminationen aufgrund des unvermeidlichen Nichtwissens ein anderer Maßstab zur Definition von Fehlern und in der Folge zur Klärung von Haftungsfragen Anwendung findet. Werden Wissensgrenzen und die Grenzen der gewählten Methoden aufgezeigt und in Dokumenten (insbes. in Verträgen und Gutachten) festgeschrieben, dann werden ungünstige Ereignisse infolge von Entscheidungen nicht als Fehler verstanden, der hätte vermieden werden können.

An dieser Stelle lassen sich Parallelen zu dem von Mary Douglas für westliche Kulturen im Umgang mit Risiken identifiziertem Prinzip der Verantwortungsfreistellung feststellen. Dieses Prinzip greift bei der Feststellung einer Schuld, sobald eine Person auf Unsicherheit und Nichtwissen verweisen kann und ist in Deutschland als Prinzip der Wahrheitsbewertung auch in zivilen Strafprozessen verankert (Douglas 1992; Hackenberg 1995). Die Klärung der Frage „Hätte der Betreffende es wissen können?‘ ist dabei zentral und ist ein wesentliches Merkmal des Umgangs mit Nichtwissen (vgl. Wehling 2011). Genaugenommen geht es also bei An- 
wendung dieses Prinzips darum, nach dem Eintreten eines ungünstigen Ereignisses zu klären, ob im entscheidenden Moment relatives oder absolutes Nichtwissen vorlag. Im Gegensatz dazu erfolgt der präzisierte Verweis auf Nichtwissen in den Fallbeispielen der Altlastensanierung vor einer Entscheidung. Auf dieser Grundlage können unerwünschte Entwicklungen als produktiver Misserfolg verstanden und als Anlass zum Lernen genommen werden, um gemeinsam Lösungsstrategien zu erarbeiten. Die Beteiligten entwickeln eine Einstellung des Vorbereitetseins.

\section{Vorbereitetsein auf kleine und große Überraschungen}

Der Austausch über die Grenzen des Wissens, der durch ein besonderes Fehlerverständnis sowie die Möglichkeit der Begründung von Nichtwissensentscheidungen begünstigt wird, führt zu einer Sensibilisierung der Akteure und einer Erwartungshaltung für unvorhergesehene Ereignisse. Wenn die Interviewpartner über die Grenzen ihres Wissens sprechen, machen sie - wie der Behördenvertreter im folgenden Zitat - diese Erwartungshaltung häufig explizit:

„, Und dann ist noch der Stich zu machen, der ist jetzt auch angefangen, zwischen der Radegaster Straße und der Planstraße A. Dort erwarten wir eigentlich noch hochkontaminiertes Material. “(Ingenieur, 2007)

Ob Behördenvertreter, Ingenieur oder auch lokale Politiker - immer wieder wird verdeutlicht, dass ein Bewusstsein über möglicherweise auftretende Altlasten besteht. Darüber hinaus wird das Wissen über die Besonderheit von Altlasten und die Notwendigkeit, grundsätzlich auf unerwartete Ereignisse eingestellt zu sein, weiteren Akteuren mitgeteilt. Dieser Wissenstransfer erfolgt z.B. mit Hilfe des verwaltungstechnischen Instruments der Auflage:

,Wir erteilen die baurechtliche Genehmigung, je nachdem, was da beantragt ist. Wir erteilen dann entsprechende Auflagen; wir kennen ja dann auch dieses Gebiet schon, wissen also schon, womit man da rechnen könnte. "(Fachbehörde, 2007)

Die Verknüpfung der Information mit einer Genehmigung erhöht die Chance, dass sie auch wahrgenommen wird. Auf die Einstellung des Vorbereitetseins wird durch die Akteure somit aktiv hingearbeitet, sowohl in der Planungsphase von Sanierungsprojekten als auch in der Durchführungsphase von Sanierungen.

Das Vorbereitetsein in der Planungsphase lässt sich in Anlehnung an die Arbeiten der Katastrophenforschung als ,preparedness“ bezeichnen. Lakoff (2007) diskutierte ,preparedness“ als Entscheidungsrationalität, die den Umgang mit Ereignissen ermöglicht, deren Eintrittszeitpunkt und Intensität sich mit den Methoden der wahrscheinlichkeitstheoretischen Abschätzung nicht bestimmen lassen. Dabei geht es nicht um die Vermeidung ungünstiger Ereignisse, sondern um die Entwicklung von Strategien zur Minimierung der Verletzbarkeit und der Stärkung der Funktions- und Widerstandsfähigkeit von Gesellschaften. Mit Blick auf eine solch langfristige Perspektive wurde in der US-amerikanischen Fallstudie ein Plan entwickelt, der den Umgang mit unvorhergesehenen Ereignissen regelt. In der Präambel heißt es:

"Although significant remediation efforts have taken place, contaminated soil or groundwater (contaminated media) remains at the former Reynolds Metals Company (RMC) facility in Troutdale, Oregon. This Contaminated Media Management Plan (CMMP) summarizes the proper management of any contaminated media in the event that they are encountered during future development of the site. This CMMP is intended to communicate." (Contaminated Media Management Plan, 2007)

Sehr deutlich wird die Aufgabe des Dokumentes - die Kommunikation und Vermittlung von Informationen - mit einem klaren Verweis auf Wissenslücken beschrieben. Adressaten sind 
in erster Linie Akteure, die zum Zeitpunkt der Sanierung nicht in das Projekt involviert waren und möglicherweise auch nicht über Erfahrungen im Umgang mit Altlasten verfügen. Der Plan stellt somit den Versuch dar, Wissen über das Nichtwissen für den Zeitraum von einigen Jahren zu erhalten und zu vermitteln. In diesem Zusammenhang bezieht sich das Vorbereitetsein recht allgemein auf die generelle Möglichkeit des Auftretens unvorhergesehener Altlastenfunde.

In der Umsetzungsphase konkreter Maßnahmen wird detaillierter definiert, worauf genau die Akteure vorbereitet sein sollen. Statt beispielsweise jeden Erdaushub auf Schadstoffe im Labor analysieren zu lassen, werden die Arbeiter dahingehend geschult, auf Veränderungen des Bodens - Geruch und Farbe - zu achten. Erst wenn eine Änderung festzustellen ist, werden detaillierte Untersuchungen veranlasst:

„[D]er Aushub [...] wird in jedem Fall beprobt. Wenn Sie in so einem Bereich sind, wo es eben von allen Seiten reinläuft, dann können Sie natürlich sagen, bis sich das ändert [das Aussehen des Aushubs], fahren wir das Zeug alles auf dieses Haufwerk. Wenn sich das ändert, sagen die Leute [die Bauarbeiter] Bescheid. " (Ingenieur, 2007)

Mit Hilfe des aus der Forschung zu hochsensiblen, soziotechnischen Systemen (sogenannten high reliability organizations) stammenden Begriffs der „awareness“ kann dieses Vorbereitetsein beschrieben werden. Awareness bezeichnet dabei die Aufmerksamkeit für schwache Signale, die Hinweise auf kleinste Abweichungen vom Normalzustand (Gerüche, Farbveränderungen etc.) geben und möglicherweise Vorboten von größeren Katastrophen sind (La Porte / Consolini 1991; Weick / Sutcliffe 2007).

Ähnlich wie Weick und Sutcliffe (2007) es für hoch sensible Systeme beschrieben, muss diese Aufmerksamkeit in der Altlastensanierung permanent gegen Routinisierungen in Arbeitsabläufen durchgesetzt werden. Ein entscheidender Unterschied zu diesen Systemen ist allerdings, dass überraschende Altlastenfunde die größere Normalität sind als der ungestörte Betriebsablauf, ja, dass der Fokus gerade auf das Finden von Abweichungen gerichtet ist. Da Altlasten (Kontaminationen und industrielle Artefakte) niemals so detailliert erkundet werden können, dass Überraschungen während der Sanierung ausgeschlossen sind, sorgen diese selbst für eine ständige Sensibilisierung bei allen Beteiligten.

Vorbereitetsein speist sich in diesem Kontext aus dem spezifizierten Nichtwissen (ignorance), speziell aus der grundsätzlichen Erkenntnis, dass es im Projektverlauf Überraschungen durch unvorhergesehene Altlasten geben wird. Das spezifizierte Nichtwissen impliziert Hypothesen über mögliche Ereignisse und Signale ihres Auftretens (z.B. Konsistenz des Bodens, unterirdische Hohlräume, Geruch des Grundwassers). Die Erwartung von Überraschungen fördert die ständige Überprüfung der vorgefundenen Situation anhand dieser Hypothesen, um Abweichungen zu identifizieren. Ereignisse, die komplett außerhalb des Erfahrungshorizontes liegen und nicht ohne technische Hilfsmittel erfahrbar sind - beispielsweise geruchs- und farblose Schadstoffe, deren Auftreten aufgrund der Vorkenntnisse über einen Standort nicht vermutet wird (z.B. radioaktive Stoffe am Standort eines Schwelwerkes) - können nur zufällig wahrgenommen werden. Solche Ereignisse sind mit der Kategorie des nicht reflektierbaren Nichtwissens (nescience) oder bei Wehling (2006) des unerkannten Nichtwissens verbunden, dessen sich Akteure nur in der Reflexion vergangener Ereignisse bewusst werden können.

Gleichwohl scheint es so, als würde es die generelle Erwartung des Unerwarteten den Akteuren ermöglichen, auch leichter mit Ereignissen jenseits des Erfahrungshorizontes umzugehen. In diesem Sinne beinhaltet Vorbereitetsein auch nicht unbedingt konkrete Maßnahmen, die vorgehalten werden, um mit eventuell eintretenden Überraschungen umzugehen. Vielmehr handelt es sich um ein Bewusstsein für die grundsätzliche Möglichkeit von Überraschungen, die deren Auftreten nicht verhindert, aber einen adäquaten Umgang mit ihnen ermöglicht. 


\section{Was tun wenn plötzlich alles anders ist? - Strategien}

Die Einstellung des Vorbereitetseins ist eine entscheidende Grundlage für die Entwicklung von Strategien (nicht von konkreten Maßnahmen), die es den beteiligten Akteuren ermöglichen, mit erwartbaren, aber im Detail unvorhergesehenen Veränderungen und Ereignissen umzugehen.

Eine erste Strategie ist die gezielte Auswahl von Akteuren, die im Wesentlichen zu Beginn eines Projektes stattfindet, aber für spezielle, sich neu ergebende Aufgaben auch im weiteren Projektverlauf erfolgt. Die Auswahlmöglichkeiten sind in einem Altlastenprojekt jedoch eingeschränkt, und zentrale Akteure wie z.B. Fachverwaltungen, aber auch die politische Gemeindevertretung durch die räumliche Organisation entsprechender Strukturen und Zuständigkeiten vorgegeben. So kann sich ein Flächeneigentümer zwar das Ingenieurbüro wählen, das die Sanierung betreut, aber kaum den zuständigen Mitarbeiter der Umweltbehörde oder den Gemeinderat, in deren Zuständigkeitsbereichen das Grundstück liegt. Darüber hinaus können Projektbeteiligte wie Ingenieurbüros, Projektentwickler oder Baufirmen frei gewählt werden. Entscheidende Kriterien zur Auswahl dieser meist institutionellen Akteure sind ihre Reputation und Erfahrung in der Altlastensanierung sowie ihre Verlässlichkeit. Darüber hinaus ist der Wille zur (fachübergreifenden) Kooperation im Projekt und über die Grenzen unterschiedlicher Institutionen hinaus von Bedeutung.

Die Analyse der Projekte machte deutlich, dass gerade der Umgang mit Nichtwissen (werden innerhalb der Binnenstruktur der Akteure z.B. Wissenslücken thematisiert oder wird eher auf vermeintlich sicheres Wissen verwiesen) und die Offenheit gegenüber unerwarteten Entwicklungen, aber auch die Geschwindigkeit der Entscheidungsfindung bei überraschenden Entwicklungen den Verlauf des Projektes beeinflussen.

Eine zweite Strategie ist die Schaffung einer Arbeitsorganisation, die einen Austausch über die ständig neu zu definierende Relevanz des Nichtwissens und ein schnelles Reagieren bei Überraschungen ermöglicht. In der Regel sind Altlastenprojekte in klar definierten hierarchischen Auftraggeber-Auftragnehmer-Verhältnissen organisiert. Obwohl die Aufgabenbereiche und Zuständigkeiten damit vorab bestimmt sind, können die Grenzen im Alltag oft fließend sein, wie die folgende Schilderung aus Weißandt-Gölzau zeigt:

„,Und das klappt immer, dass der [Bauleiter Straßenbau] dann auch mal mit [zu den Sanierungsmaßnahmen] hinguckt. Und wir [Sanierungsbüro] gucken auch mal nach Herrn [Bauleiter Straßenbau]s Sachen, so dass man sich mal gegenseitig unterstützt. Das funktioniert auch mit einer guten Zusammenarbeit der Bauleiter. " (Ingenieur, 2008)

Die Bereitschaft zur Kommunikation über vertragliche Bindungen und definierte Aufgabenbereiche hinaus schafft Möglichkeiten, Informationen über neue Entwicklungen schnell auszutauschen, frühzeitig Hinweisen auf unvorhergesehene Entwicklungen (im Sinne schwacher Signale) nachzugehen und - wenn notwendig - die Relevanz von Nichtwissen neu zu bestimmen. Diese Arbeitsorganisation ermöglicht auch, dass innerhalb der hierarchischen Organisationsstruktur temporär nicht-hierarchisch strukturierte Interaktionen stattfinden.

Die Bedeutung, die der Kommunikation zwischen den Projektpartnern und auch der Einigkeit über Entscheidungsregeln und Arbeitsabläufe beigemessen wird, wird im folgenden Zitat zur Sanierung in Troutdale deutlich:

"So they [responsible enterprise] actual even brought in meeting facilitators and we spent a day and a half very early on [...] Meeting facilitators who work at developing a common agreement on how you will conduct your work and how you will move forward, how you will make decisions and so forth." (Bundesbehörde, 2010) 
Unabhängig von konkreten Fragen des Sanierungsprojektes wurden Ressourcen darauf verwendet die Grundzüge der Arbeitskooperation zu klären, auf die sich in kritischen Momenten des Projektes immer berufen werden konnte.

Teil einer solchen Arbeitsorganisation ist auch die Schaffung formeller Institutionen. $\mathrm{Zu}$ diesen gehören insbesondere regelmäßige Treffen aller Projektpartner, in denen neue Entwicklungen vorgestellt und Lösungsansätze für auftretende Probleme diskutiert und die Relevanz des Nichtwissens thematisiert werden. In Phasen großer Dynamik und zahlreicher Überraschungen können solche Treffen wöchentlich stattfinden. Im Projekt in Weißandt-Gölzau wurde eine weitere Institution geschaffen - ein Vor-Ort-Büro der Mitarbeiter des Projektkoordinators. Es diente nicht nur den Projektpartnern als lokale Anlaufstelle für den Informationsaustausch (Organisation der Informationsflüsse), sondern bildete auch die permanente Anlaufstelle für Akteure, die nicht direkt in das Sanierungsgeschehen involviert, aber unter Umständen von Begleiterscheinungen betroffen waren (z.B. Anwohner).

Eine dritte Strategie ist schließlich der gezielte Einsatz rechtlicher Instrumente, um die Tatsache des Nichtwissens explizit einzubeziehen und um Fragen der Haftung im Vorhinein zu klären. Sie werden als passendes Instrument verstanden, um die Vertragspartner auf die Notwendigkeit einer Haltung des Vorbereitetseins aufmerksam zu machen.

„Das heißt, dass man bestimmte Auflagen, die man jetzt nicht formuliert hat, weil man die Umstände gar nicht so im Detail erfassen kann, dass man sich also als Behörde die Möglichkeit offen lässt, dort nachträglich noch Auflagen nachzuschieben. " (Fachbehörde, 2007)

Wie hier im Zitat zum Ausdruck gebracht, wird insbesondere mit flexiblen Rechtsmitteln wie dem Auflagenvorbehalt (§ 36 Abs. 2 Nr. 5 Verwaltungsverfahrensgesetz), dem Nachtragsangebot oder speziellen Vertragsklauseln gearbeitet, die es situativ ermöglichen Anpassungen vorzunehmen und damit auf veränderte Rahmenbedingungen zu reagieren.

\section{Schlussbemerkung - Nichtwissen aktiv einbeziehen}

Ausgangspunkt der vorgestellten Analysen war die Feststellung, dass bei Entscheidungen zur Umweltgestaltung oftmals nicht auf vollständiges Wissen über Prozesse von Ökosystemen zurückgegriffen werden kann. Während es das Alltagsgeschäft von wissenschaftlicher Wissensproduktion ist, Nichtwissen mit dem Ziel zu identifizieren, neue Erkenntnisse zu generieren und dabei auch Nichtwissensansprüche zu verhandeln, stellte sich die Frage, wie im Alltag der Umweltgestaltung mit Nichtwissen in Entscheidungsprozessen umgegangen wird.

In der Altlastensanierung, einem wichtigen Bereich des nachsorgenden Umweltschutzes, ist es nicht möglich, Entscheidungen in jedem Fall auf Grundlage vollständigen Wissens zu treffen. Die permanente Auseinandersetzung mit immer neuen benennbaren Wissenslücken und unerwarteten Entwicklungen ist zentral und wird geradezu zur Normalität. Das bedeutet weder, dass alle Altlastensanierungen konfliktfrei verlaufen, noch heißt es, dass nicht lange nach Abschluss entsprechender Projekte Konflikte auftauchen können. In zwei konkreten Beispielen der Sanierung kontaminierter Flächen zeigte sich jedoch: wenn sich die Beteiligten grundsätzlich über das Ziel - die Notwendigkeit der Beseitigung von Kontaminationen - einig sind, kann eine gemeinsame, offene Auseinandersetzung mit Nichtwissen stattfinden und können kreative Lösungen entwickelt werden. Dies ermöglichte es, der Frage nach Nichtwissensentscheidungen exemplarisch nachzugehen, und erste Hinweise für ähnliche Analysen in anderen Feldern der Umweltgestaltung zu liefern. Das unvollständige Wissen in Entscheidungsmomenten führte in den Fallbeispielen nicht, wie erwartet werden könnte, zu Handlungsunsicherheiten. Vielmehr zeigte die Analyse von Entscheidungsprozessen mit Hilfe dynamischer Nichtwissensbegriffe, dass sich Akteure ihres Nichtwissens bewusst sind und in einer aktiven 
Auseinandersetzung entscheiden können, welche Handlungsrelevanz ihm zukommt. Die Neubestimmung der Relevanz des Nichtwissens ist ein fortlaufender Prozess, da überraschende Ereignisse und neue Erkenntnisse sie erneut in Frage stellen können.

Stets haben die einzelnen Akteure unterschiedliche Möglichkeiten und Interessen, Wissensgrenzen zu benennen. So können Experten aufgrund ihrer fachlichen Kenntnisse Nichtwissen thematisieren, das dann Gegenstand der Verhandlung wird. Victoria Wibeck (2009) bezeichnete diese Form der Kommunikation über Unsicherheiten als das Integrationsmodell in der Umwelt-Kommunikation: Wichtige Reflexions- und Lernprozesse können angestoßen werden, wenn Experten ihre Annahmen über Wissensgrenzen transparent machen und mit Laien teilen, statt bereits gefestigte Interpretationen weiter zu geben. Entsprechende Lernprozesse wurden in den Fallstudien hinsichtlich der Begründungsmuster von Nichtwissensentscheidungen und dem Verständnis von Fehlern deutlich. Auch die Entwicklung einer Einstellung des Vorbereitetseins für unvorhergesehene Ereignisse ist als Ergebnis eines solchen Prozesses $\mathrm{zu}$ verstehen. Insbesondere die aufgrund der aktiven Einbeziehung von Nichtwissen entwickelte Strategie Wissenslücken zu dokumentieren und öffentlich zugänglich zu machen, scheint jedoch das Potenzial zu haben, künftige Konflikte zu vermeiden. Auf dieser Basis schufen die Akteure in den untersuchten Sanierungsprojekten einen Handlungsrahmen für ihre Arbeit, bestehend aus einer flexiblen Arbeitsorganisation mit klarer Aufgabenteilung, spezifischen Institutionen zur Erleichterung der Kommunikation sowie flexiblen rechtlichen Instrumenten. Dieses spezifische und teilweise kreative Arrangement ermöglichte es, Nichtwissen zu thematisieren und bewusste Nichtwissensentscheidungen zu treffen, und weist damit Ähnlichkeiten zu dem auf, was an anderer Stelle als Realexperiment beschrieben wurde (Gross et al. 2003).

Wie die analysierten Beispiele der Altlastensanierung zeigen, ist die gezielte Schaffung eines Rahmens, der einen undramatischen Umgang mit Nichtwissen erlaubt, für einen konkreten zeitlichen (Dauer des Projektes) und einen spezifischen räumlichen Kontext (z.B. ein konkretes Sanierungsprojekt) möglich. Förderlich ist dabei sicherlich, dass Altlastensanierungen häufig in ihren räumlichen Wirkungen einzugrenzen sind, und dass es sich zudem um ein aktuell vergleichsweise wenig beachteten Bereich des nachsorgenden Umweltschutzes handelt. Diese Situation erleichtert sicherlich einen vergleichsweise offenen Umgang mit unbeantworteten Fragen. Das Aufspüren ähnlicher Handlungsrahmen in anderen Bereichen der Umweltgestaltung bleibt die Aufgabe künftiger Forschungen. Nichtwissen bietet sich dabei als analytische Perspektive an. Statt die Aufmerksamkeit ausschließlich auf die Vermeidung möglicher nicht intendierter Gefährdungen in Folge von Entscheidungen zu lenken oder die unbedingte Schaffung umfassenden Wissens vor einer Entscheidung zu fokussieren, wird es so möglich, innovative Strategien im Umgang mit nicht zu beantwortenden Fragen zu identifizieren. Dabei können vielfältigere Strategien als die bloße Wissensbeschaffung (diese aber auch!) den alltäglichen Umgang mit Nichtwissen prägen. Sollten ähnliche Muster und Strategien auch in anderen Bereichen der Umweltgestaltung zu finden sein, könnte perspektivisch eine Pluralisierung von Strategien im Umgang mit Nichtwissen erreicht werden (Böschen / Wehling 2004).

\section{Literaturverzeichnis}

Beck, U. / A. Giddens / S. Lash (Hrsg.) (1996): Reflexive Modernisierung. Eine Kontroverse, Frankfurt / Main.

Böschen, S. / P. Wehling (2004): Wissenschaft zwischen Folgenverantwortung und Nichtwissen. Aktuelle Perspektiven der Wissenschaftsforschung, Wiesbaden.

Brandt, E. (1993): Einleitung, in: Ders. (Hrsg.), Altlasten. Bewertung. Sanierung. Finanzierung, 3. Auflage, Taunusstein, S. 14-20.

Collingridge, D. (1980): The Social Control of Technology, London. 
Collins, M.H. / R. Evans (2002): The Third Wave of Science Studies: Studies of Expertise and Experience, in: Social Studies of Science 32, S. 235-296.

De Sousa, C. (2008): Brownfields Redevelopment and the Quest for Sustainability, Oxford.

Dixon, T. / M. Raco / P. Catney / D.N. Lerner (Hrsg.) (2007): Sustainable Brownfield Regeneration. Livable Places form Problem Spaces, Oxford.

Douglas, M. (1992): Risk and Blame: Essays in Cultural Theory, London.

Dovers S. / T. Norton / J. Handmer (2001): Ignorance, Uncertainty and Ecology: Key Themes, in: J. Handmer / T. Norton / S. Dovers (Hrsg.), Ecology, Uncertainty and Policy. Managing Ecosystems for Sustainability, London, S. 1-25.

Faber, M. / J.L.R. Proops / R. Manstetten (1993): Evolution, Time, Production and the Environment, Berlin.

Franzius, V. / N. Altenbockum / G. Thomas (2009): Handbuch Altlastensanierung und Flächenmanagement. 20 Jahre - 20 Fragen - 20 Persönlichkeiten, Heidelberg.

Frickel, S. / S. Gibbon / J. Howard / J. Kempner / G. Ottinger / D.J. Hess (2010): Undone Science: Charting Social Movement and Civil Society Challenges to Research Agenda Setting, in: Science, Technology \& Human Values 35, S. 444-473.

Funtowicz, S.O. / J.R. Ravetz (1993): The Emergence of Post-Normal Science, in: R. von Schomberg (Hrsg.), Science, Politics, and Morality: Scientific Uncertainty and Decision Making, Dordrecht, S. 85-123.

Gibbons, M. / H. Nowotny / C. Limoges / S. Schwartzman / P. Scott / M. Trow (1994): The new Production of Knowledge: The Dynamics of Science and Research in Contemporary Societies, London.

Green, J. (2009): Is it Time for the Sociology of Health to Abandon 'Risk'?, in: Health, Risk \& Society 11, S. 493-508.

Gross, M. (2007): Communicating Ignorance and the Development of Post-Mining Landscapes, in: Science Communication 29, S. 264-270.

Gross, M. (2010): Ignorance and Surprise. Science, Society, and Ecological Design, Cambridge.

Gross, M. (2012): 'Objective Culture' and the Development of Nonknowledge: Georg Simmel and the Reverse Side of Knowing, in: Cultural Sociology 6, im Erscheinen.

Gross, M. / H. Hoffmann-Riem / W. Krohn (2003): Realexperimente: Robustheit und Dynamik ökologischer Gestaltungen in der Wissensgesellschaft, in: Soziale Welt, 54, S. 241-257.

Hackenberg, W. (1995): Die Erklärung mit Nichtwissen (x138 IV ZPO). Zugleich eine kritische Analyse der Lehre der ,allgemeinen Aufklärungspflicht“, Berlin.

Hess, D.J. (2009): The Potentials and Limitations of Civil Society Research: Getting Undone Science Done, in: Sociological Inquiry 79, S. 306-327.

Hofer, H. (2009): Handlung und Legitimation im Zuge reflexiver Modernisierung, in: F. Böhle / M. Weihrich (Hrsg.), Handeln unter Unsicherheit, Wiesbaden, S. 139-147.

Janich, N. / L. Rhein / A. Simmerling (2010): "Do I know what I don't know?" The Communication of Non-Knowledge and Uncertain Knowledge in Science, in: Fachsprache. International Journal of Specialized Communication, S. 86-99.

Kewell, B. (2010): Heteroglossic Representations of Scientific Uncertainty: A Sociolinguistic Analysis of Expert Witness Testimony to the Bristol Inquiry, in: Science Technology Human Values 36, S. 816-841.

de Krom, M. P.M.M. / P. Oosterveer (2010): Contesting Risk and Responsibility: European Debates on Food and Agricultural Governance of Avian Influenza. Nature and Culture 5, S. 175-195.

Kuckartz, U. (2007): Einführung in die computergestützte Analyse qualitativer Daten, Wiesbaden.

La Porte, T.R. / P.M. Consolini (1991): Working in Practice But Not in Theory: Theoretical Challenges of "High Reliability Organizations", in: Journal of Public Administration Research and Theory 1, S. 19-47.

Lakoff, A. (2007): Preparing for the Next Emergency, in: Public Culture 19, S. 247-271. 
Lau, C. (1989): Risikodiskurse: Gesellschaftliche Auseinandersetzungen um die Definition von Risiken, in: Soziale Welt 40, S. 418-436.

Lau, R.W.K. (2009): The Contemporary Culture of Blame and the Fetishization of the Modernist Mentality, in: Current Sociology 57, S. 661-683.

Lidskog, R. (2011): Regulating Nature: Public Understanding and Moral Reasoning. Nature and Culture 6: S. 149-167.

McGoey, L. (2007): On the Will to Ignorance in Bureaucracy, in: Economy and Society 36, S. 212-235.

Merton, R.K. (1987): Three Fragments from a Sociologist's Notebook: Establishing the Phenomenon, Specified Ignorance, and Strategic research Materials, in: Annual Review of Sociology 13, S. 1-28.

Moore, W. / M. Tumin (1949): Some Social Functions of Ignorance, in: American Sociological Review 14, S. 787-795.

Powell, M.C. / M. Colin (2009): Participatory Paradoxes. Facilitating Citizen Engagement in Science and Technology From the Top-Down? in: Bulletin of Science Technology Society 29, S. 325-342.

Proctor, R.N. (1995): Cancer Wars: How Politics Shapes What We Know \& Don't Know About Cancer, New York / NY.

Ravetz, J.R. 1993: The Sin of Science : Ignorance of Ignorance, in: Science Communication 15, S. 157-181.

Smithson, M. (1993): Ignorance and Science: Dilemmas, Perspectives, and Prospects, in: Science Communication, 15, S. 133-156.

Smithson, M. (1985): Towards a Social Theory of Ignorance, in: Journal for the Theory of Social Behaviour, 15, S. 151-172.

Simmel, G. (1908): Soziologie. Untersuchungen über die Formen der Vergesellschaftung, Berlin.

Stocking, S.H. (1998): On Drawing Attention to Ignorance, in: Science Communication 20, S. 165-178.

Stocking, S.H. / L.W. Holstein (1993): Constructing and Reconstructing Scientific Ignorance. Ignorance Claims in Science and Journalism, in: Science Communication 15, S. 186-210.

Strauss, A. / J. Corbin (1990): Basics of Qualitative Research. Grounded Theory Procedures and Techniques, Thousand Oaks / CA.

Strübing, J. (2008): Grounded Theory. Zur sozialtheoretischen und epistemologischen Fundierung des Verfahrens der empirisch begründeten Theoriebildung, Opladen.

Steyaert, P. / J. Jiggins (2007): Governance of Complex Environmental Situations through Social Learning: A Synthesis of SLIM's Lessons for Research, Policy and Practice, in: Environmental Science \& Policy 10, S. 575-586.

Wehling, P. (2006): Im Schatten des Wissens? Perspektiven der Soziologie des Nichtwissens, Konstanz.

Wehling, P. (2011): Vom Risikokalkül zur Governance des Nichtwissens. Öffentliche Wahrnehmung und soziologische Deutung von Umweltgefährdungen, in: M. Groß (Hrsg.), Handbuch Umweltsoziologie, Wiesbaden, S. 529-548.

Weick, K.E. / K.M. Sutcliffe (2007): Managing the Unexpected: Resilient Performance in an Age of Uncertainty, San Francisco / CA.

Weingart, P. / M. Carrier / W. Krohn (2007): Experten und Expertise, in: Dies. (Hrsg.), Nachrichten aus der Wissensgesellschaft. Analysen zur Veränderung der Wissenschaft, Weilerswist, S. 293-304.

Wibeck, V. (2009): Communicating Uncertainty: Models of Communication and the Role of Science in Assessing Progress towards Environmental Objectives, in: Journal of Environmental Policy and Planning 11, S. 87-102.

Wildavsky, A. (1988): Searching for Safety, Piscataway / NJ. 
Dr. Alena Bleicher Helmholtz-Zentrum für Umweltforschung GmbH - UFZ Permoserstr. 15 04318 Leipzig alena.bleicher@ufz.de 